Editorial

\title{
Management of Cytomegalovirus (CMV) in Hematopoietic Stem Cell Transplant (HSCT)
}

\author{
Volume I Issue 4 - 2014 \\ Yasser E Elborai ${ }^{1,2}$ \\ 'Department of Pediatric Oncology, National Cancer Institute, \\ Cairo University, Egypt \\ ${ }^{2}$ Department of Pediatric Hematology \& Oncology, Prince \\ Sultan Military Medical City, Saudi Arabia
}

\section{Introduction}

Allogeneic hematopoietic cell transplant (HCT) recipients are at increased risk for a variety of infections based upon their degree of immunosuppression and exposures. Autologous HCT recipients are also at increased risk for infection, although to a lesser degree than those who have undergone allogeneic HCT. The types of infections to which these hosts are most vulnerable can be roughly divided based upon the approximate time elapsed since transplantation.

Cytomegalovirus (CMV) is a member of the Herpes virus family with a genome of double-stranded linear DNA. ${ }^{1}$ Although most CMV infections in immunocompetent patients are asymptomatic or cause mild disease, the virus can cause serious disease in newborns and immunocompromised children. ${ }^{2} \mathrm{CMV}$ stays in lymphocytes forever and may reactivate when patients are immunocompromised.

\section{Clinical presentation of CMV diseases}

CMV reactivation is defined as positive CMV-PCR > 500 copies, while CMV disease is defined as CMV related dysfunction in different organs. GI disease is the most common manifestation and can escape blood-based surveillance by PCR and pp65 antigenemia assay in approximately $25 \%$. Pneumonia and interstitial pneumonitis is the most serious manifestation but is now infrequent because of current prevention strategy. ${ }^{3}$ Hepatitis, retinitis and encephalitis are serious complication of CMV infection. Immunosuppression and myelosuppression can cause decreased counts and immunosuppression may also lead to bacteremia and fungemia. CMV reactivation or infection may trigger GVHD and on the other hand, immunosuppression for GVHD may increase CMV, a vicious circle. ${ }^{4}$ There is some recent evidence correlating CMV reactivation with decreased rate of relapse in leukemic patients but data is not conclusive. ${ }^{5,6}$

\section{Choosing the donor according to CMV status}

CMV status is one of many factors, which can determine the donor, but it is not the most important factor. Matching at HLA-A, $\mathrm{B}$, DR is more important than CMV status, while CMV status may be more important than matching at HLA-C, DQ and DP. And also, CMV status is more important than donor age or blood group match. The best clinical situation is CMV negative donor for CMV negative patient. If the patient is CMV positive, the donor should be CMV positive to allow adoptive transfer of donor immunity. ${ }^{4}$ If the patient is CMV negative and there is no available CMV negative donor, we can choose CMV positive donor. The worst clinical scenario is choosing CMV negative donor for CMV positive patient.

\section{Pre-transplant screening for CMV}

The pre-transplantation screening is designed to prevent posttransplant infection by assessing patient and donor status. Within 30 days of admission, we should check donor's CMV IgG titer to

\begin{abstract}
Correspondence: Yasser E Elborai, Department of Pediatric Oncology, National Cancer Institute, Cairo University, Kornish El-Nile - Fom El- Khaleg, I 1796 Cairo, Egypt, Tel 967000000000 , Email yasserborai@hotmail.com
\end{abstract}

Received: August 01, 2014 | Published: August 02, 2014

assess past infection. Also, we should check patient's CMV IgG titer to assess past infection, and CMV PCR to rule out current viremia.

\section{Risk factors for CMV reactivation}

Many factors can attribute in CMV reactivation; transplantation from a CMV-negative donor to a CMV-positive recipient and transplantation with CMV viremia or an increased CMV viral load are the most important factors that can cause CMV reactivation. Other factors that contribute in CMV reactivations are low CD4 T-cell count post SCT, undetectable CMV specific T-cell immunity, use of anti T-cell agents (ATG), T-cell depletion of the graft and cord blood donor.

\section{Pre-engraftment management of CMV in HSCT}

If the recipient is CMV antibody negative and donor is CMV antibody negative or positive, no specific CMV prophylaxis is used. While, if the recipient is CMV antibody positive and donor is CMV antibody negative or positive, prophylaxis with Acyclovir may be needed; if the recipient $<0.6 \mathrm{~m} 2$ : Acyclovir $15 \mathrm{mg} / \mathrm{kg}$ IV Q8hr d-1 through $\mathrm{d}+30$ but if the recipient $>/=0.6 \mathrm{~m} 2$ : Acyclovir $500 \mathrm{mg} / \mathrm{m} 2 \mathrm{IV}$ Q8hr d-1 through d+30.7

\section{Post-engraftment Management of CMV in HSCT}

If the recipient and donor are CMV Antibody negative pretransplant, no routine screening is needed and we will send for CMV PCR if clinical symptoms or signs indicate CMV infection and no specific CMV prophylaxis is used. While if the recipient is CMV Antibody positive and/or donor CMV antibody positive pretransplant, weekly screening for CMV reactivation consisting of CMV blood PCR test should be done. Screening should begin at $\mathrm{d}+21$ or at engraftment if earlier and continue weekly through $\mathrm{d}+100$ or $\mathrm{d}+120$ if prior to CMV reactivation. Screening may be continued beyond $\mathrm{d}+100$ for patients at particular risk of CMV e.g. GVHD or clinical judgment. If screening is negative, no treatment and continue 
screening is needed every week while if CMV reactivation occurs, anti-viral therapy will be initiated.

Ganciclovir with therapeutic dose $(5 \mathrm{mg} / \mathrm{kg}$ per dose IV every 12hours) given for 14days and/or until CMV reactivation resolves may lead to Dose-dependent bone marrow suppression which is reversible. Thus, CBC should be closely monitored. Also nausea, GI upset, increase creatinin and renal insufficiency may occur. Thus, dose should be adjusted in renal insufficiency or renal failure. IVIG or CMV IgG can be given 3 times a week for 2 weeks then weekly for $\mathrm{d}+100$. If clinical or virologic response is not seen or if neutropenia occurs, then Foscarnet $(60 \mathrm{mg} / \mathrm{kg}$ IV q8h) replaces Gancyclovir if renal function allows.

\section{New treatment options for CMV}

Lipid Cidofovir (CMX-001) and non-nucleoside inhibitor (AIC246) are novel drugs currently undergoing phase 2 trials in HSCT setting. ${ }^{8,9}$ Keratinocyte growth factor \& IL7 are non-specific strategies and recent studies showed promising results. ${ }^{10}$ Maribavir is a novel DNA synthesis inhibitor that is being evaluated for use against CMV because it does not cause myelosuppression. The phase 3 trial failed to reduce the incidence of CMV disease and showed only a modest antiviral effect. ${ }^{11}$ Maribavir in high dose may be beneficial in resistant cases. ${ }^{3}$ Immune augmentation using $\mathrm{T}$ cell transfusion and Active immunization $\mathrm{T}$ cell transfusion means transfer of donor derived CMV specific T-cells that have been reported without significant toxicity and antiviral effect in some small series of patients with refractory CMV viremia or disease. ${ }^{10}$ Active immunization using DNA vaccines are also being studied in HSCT recipients. ${ }^{12}$

\section{Acknowledgements}

None.

\section{Conflicts of interest}

Author declares there are no conflicts of interest.

\section{Funding}

None.

\section{References}

1. Mocarski ES, Kemble GW. Recombinant cytomegaloviruses for study of replication and pathogenesis. Intervirology. 1996;39(5-6):320-330.

2. Demmler-Harrison GJ. Congenital cytomegalovirus: Public health action towards awareness, prevention, and treatment. J Clin Virol. 2009;46(Suppl 4):S1-S5.

3. Marty FM, Boeckh M. Maribavir and human cytomegalovirus-what happened in the clinical trials and why might the drug have failed? Curr Opin Virol. 2011;1(6):555-562.

4. Boeckh M, Ljungman P. How we treat cytomegalovirus in hematopoietic cell transplant recipients. Blood. 2009;113(23):5711-5719.

5. Erard V, Guthrie KA, Riddell S, et al. Impact of HLA A2 and cytomegalovirus serostatus on outcomes in patients with leukemia following matched-sibling myeloablative allogeneic hematopoietic cell transplantation. Haematologica. 2006;91(10):1377-1383.

6. Travi G, Pergam SA, Xie H, et al. Donor CMV serostatus not predictive of relapse in D-/R- pediatric HCT. Biol Blood Marrow Transplant. 2009;15(6):758-760.

7. Meyers JD. Prevention and treatment of cytomegalovirus infection after marrow transplantation. Bone Marrow Transplant. 1988;3(2):95-104.

8. Prichard MN, Kern ER, Hartline CB, et al. CMX001 potentiates the efficacy of acyclovir in herpes simplex virus infections. Antimicrob Agents Chemother. 2011;55(10):4728-4734.

9. Kaul DR, Stoelben S, Cober E, et al. First report of successful treatment of multidrug-resistant cytomegalovirus disease with the novel antiCMV compound AIC246. Am J Transplant. 2011;11(5):1079-1084.

10. Feuchtinger T, Opherk K, Bethge WA, et al. Adoptive transfer of pp65specific $\mathrm{T}$ cells for the treatment of chemorefractory cytomegalovirus disease or reactivation after haploidentical and matched unrelated stem cell transplantation. Blood. 2010;116(20):4360-4367.

11. Winston DJ, Young JA, Pullarkat V, et al. Maribavir prophylaxis for prevention of cytomegalovirus infection in allogeneic stem cell transplant recipients: a multicenter, randomized, double-blind, placebocontrolled, dose-ranging study. Blood. 2008;111(11):5403-5410.

12. Tomblyn M, Chiller T, Einsele H, et al. Guidelines for preventing infectious complications among hematopoietic cell transplant recipients: a global perspective. Preface. Bone Marrow Transplant. 2009;44(8):453-455. 